\title{
Correction to: Genetic Analysis of Hereditary Ataxias in Peru Identifies SCA10 Families with Incomplete Penetrance
}

\author{
Mario Cornejo-Olivas ${ }^{1,2}$ (D) Miguel Inca-Martinez ${ }^{1,3}$ - Raphael Machado Castilhos ${ }^{4,5}$. Gabriel Vasata Furtado ${ }^{4,6}$. \\ Eduardo Preusser Mattos ${ }^{4,6}$. Giovana Bavia Bampi ${ }^{4,6}$. Sandra Leistner-Segal ${ }^{7}$ - Victoria Marca ${ }^{1}$. Pilar Mazzetti ${ }^{1}$. \\ Maria Luiza Saraiva-Pereira ${ }^{4,6,7,8,10}$ • Laura Bannach Jardim ${ }^{4,6,7,9,10}$ • on behalf of Rede Neurogenetica
}

Published online: 15 January 2020

(C) Springer Science+Business Media, LLC, part of Springer Nature 2020

\section{Correction to Cerebellum \\ https://doi.org/10.1007/s12311-019-01098-2}

The original version of this article unfortunately contained some mistakes in Table 2. The additional row (just above SCA2) with the following information "SCA1, 1(1), 1, 50, $74,24,46$ and $0 / 1$ " should be inserted.

The original article has been corrected.

The online version of the original article can be found at https://doi.org/ 10.1007/s12311-019-01098-2

Mario Cornejo-Olivas

mario.cornejo.o@incngen.org.pe

1 Neurogenetics Research Center, Instituto Nacional de Ciencias, Neurológicas, 1271 Ancash St, Barrios Altos, 15003 Lima, Peru

2 Center for Global Health, Universidad Peruana Cayetano Heredia, Lima, Peru

3 Lerner Research Institute, Genomic Medicine, Cleveland Clinic Foundation, Cleveland, OH, USA

4 Programa de Pós-Graduação em Genética e Biologia Molecular, Universidade Federal do Rio Grande do Sul, Porto Alegre, Brazil
Serviço de Neurologia, Hospital de Clínicas de Porto Alegre, Porto Alegre, Brazil

6 Laboratório de Identificação Genética, Hospital de Clínicas de Porto Alegre, Porto Alegre, Brazil

7 Serviço de Genética Médica, Hospital de Clínicas de Porto Alegre, Porto Alegre, Brazil

8 Departamento de Bioquímica, Universidade Federal do Rio Grande do Sul, Porto Alegre, Brazil

9 Departamento de Medicina Interna, Universidade Federal do Rio Grande do Sul, Porto Alegre, Brazil

10 Instituto de Genética Médica Populacional (INAGEMP), Porto Alegre, Brazil 